\title{
Estratégias utilizadas para a avaliação da qualidade da assistência de enfermagem:
}

\section{Revisão Integrativa}

\author{
Strategies used to assess the quality of nursing care: Integrative Review \\ Estrategias utilizadas para evaluar la calidad de la atención de enfermería: Revisión Integradora
}

Recebido: 04/02/2021 | Revisado: 12/02/2021 | Aceito: 15/02/2021 | Publicado: 23/02/2021

\author{
Luiz Fernando da Silva \\ ORCID: https://orcid.org/0000-0001-7477-1324 \\ Universidade Estadual de Londrina, Brasil \\ E-mail: Luiz_fernando.silva@hotmail.com \\ Raquel Gvozd Costa \\ ORCID: https://orcid.org/0000-0002-5816-8215 \\ Universidade Estadual de Londrina, Brasil \\ E-mail: raquelgvozd@yahoo.com.br
}

\begin{abstract}
Resumo
A avaliação da qualidade da assistência de Enfermagem é um dos principais componentes que permitem que o gestor identifique as fragilidades do processo de trabalho e tome decisões. O objetivo deste trabalho foi apresentar evidências científicas sobre as estratégias utilizadas para a avaliação da qualidade da assistência de Enfermagem no contexto hospitalar. O trabalho foi realizado de acordo com protocolo estabelecido e preconizado pelo Joanna Briggs Institute: 1) Formulação da questão de pesquisa; 2) especificação dos métodos de seleção dos estudos; 3) procedimento de extração dos dados; 4) análise crítica e avaliação dos estudos incluídos 5) extração de dados e 6) apresentação da revisão integrativa do conhecimento produzido/publicado e conduzido conforme a metodologia Preferred Reporting Items for Systematic Reviews and Meta-Analyses. Observou-se que não houve aumento no número de publicações sobre o desenvolvimento e atualização de ferramentas, entre as ferramentas utilizadas, houve a predominância de questionários e todos eles avaliaram os aspectos relacionados a satisfação dos pacientes com a assistência de enfermagem, as características do processo de cuidado, os aspectos de intervenções de enfermagem e os aspectos relacionados às reclamações dos pacientes. Apesar de importantes, as ferramentas de avaliação da qualidade da assistência ainda são pouco estudadas e necessita de mais estudos na área de Enfermagem.
\end{abstract}

Palavras-chave: Qualidade da assistência à saúde; Avaliação da qualidade; Cuidado de enfermagem.

\begin{abstract}
The evaluation of the quality of nursing care is one of the main components that allow the manager to identify the weaknesses of the work process and make decisions. The aim of this study was to present scientific evidence on the strategies used to assess the quality of nursing care in the hospital context. The work was carried out according to the protocol established and recommended by the Joanna Briggs Institute: 1) Formulation of the research question; 2) specification of study selection methods; 3) data extraction procedure; 4) critical analysis and evaluation of the included studies 5) data extraction and 6) presentation of the integrative review of the knowledge produced / published and conducted according to the Preferred Reporting Items for Systematic Reviews and Meta-Analyzes methodology. It was observed that there was no increase in the number of publications on the development and updating of tools, among the tools used, there was a predominance of questionnaires and all of them evaluated aspects related to patient satisfaction with nursing care, the characteristics of the process of care, aspects of nursing interventions and aspects related to patient complaints. Although important, the tools for assessing the quality of care are still poorly studied and need further studies in the area of Nursing.
\end{abstract}

Keywords: Quality of health care; Quality evaluation; Nursing care.

\section{Resumen}

La evaluación de la calidad de la atención de enfermería es uno de los componentes principales que permiten al gerente identificar las debilidades del proceso de trabajo y tomar decisiones. El objetivo de este estudio fue presentar evidencia científica sobre las estrategias utilizadas para evaluar la calidad de la atención de enfermería en el contexto hospitalario. El trabajo se llevó a cabo según el protocolo establecido y recomendado por el Instituto Joanna Briggs: 1) Formulación de la pregunta de investigación; 2) especificación de los métodos de selección de estudios; 3 ) procedimiento de extracción de datos; 4) análisis crítico y evaluación de los estudios incluidos 5) extracción de datos y 6) presentación de la revisión integradora del conocimiento producido / publicado y realizado de acuerdo con la metodología Preferred Reporting Items for Systematic Reviews and Meta-Analyses. Se observó que no hubo incremento en el número de publicaciones sobre el desarrollo y actualización de herramientas, entre las herramientas 
utilizadas, hubo predominio de cuestionarios y todos ellos evaluaron aspectos relacionados con la satisfacción del paciente con los cuidados de enfermería, las características de la proceso de atención, aspectos de las intervenciones de enfermería y aspectos relacionados con las quejas del paciente. Aunque importantes, las herramientas para evaluar la calidad de la atención todavía están poco estudiadas y necesitan más estudios en el área de Enfermería.

Palabras clave: Calidad de la asistencia sanitaria; Evaluación de calidad; Cuidado de enfermera.

\section{Introdução}

Com os avanços tecnológicos, aumento de custo e competitividade das instituições, cresce a demanda pela melhoria na qualidade dos serviços de saúde (Hovenga \& Lowe, 2020). Sendo o foco das discussões nos serviços de saúde e nas atividades da Enfermagem, a qualidade deve ser compreendida como a probabilidade de a prestação de serviços levar ao conforto e bem-estar do paciente. Ela está intimamente ligada a vários fatores organizacionais e culturais das instituições, como: a informática, as tecnologias, a utilização da mentalidade empreendedora dos colaboradores, a prática profissional, os padrões, a educação e a ética (Hovenga \& Lowe, 2020).

A assistência de enfermagem é um dos principais componentes dos serviços de saúde e está diretamente relacionado a qualidade da instituição, bem como, a satisfação ou insatisfação do cliente (Karaca \& Durna, 2018). Quando a assistência de enfermagem é oferecida de forma inadequada, leva a consequências catastróficas. Além de experimentar angústia e insatisfação, os pacientes vivenciam a assistência de enfermagem de forma negativa e são expostos a riscos desnecessários (Richards et al., 2018). Devido a isso, apesar da importância da qualidade para os serviços de saúde, o que ainda se observa é a assistência de enfermagem inadequada ou incompleta como uma das principais responsáveis pelos desfechos negativos na evolução dos pacientes, entre eles, a morte (Recio-Saucedo, 2018).

Os gestores e líderes das instituições são fundamentais na busca pela qualidade (Hovenga \& Lowe, 2020). Para que isso ocorra de forma adequada, é necessário que eles conheçam e implementem as estratégias para avaliação da qualidade da assistência (Hovenga \& Lowe, 2020). As ferramentas de avaliação da qualidade da assistência permitem que o gestor identifique as fragilidades do processo de trabalho e tome decisões que podem melhorar a qualidade constantemente em todos os períodos de atendimento (Karaca \& Durna, 2018).

Após análise de publicações referentes às contribuições da qualidade para a enfermagem brasileira, foram identificadas três categorias temáticas: avaliação dos padrões de qualidade dos serviços de enfermagem, ações de enfermagem na implantação e / ou implantação de programas de qualidade e uso da qualidade na organização dos serviços de saúde. Nenhuma das temáticas identificadas abordam especificamente as ferramentas de avaliação (Rocha, Trevizan, Júnior \& Castelhano-Souza, 2013).

Diante do exposto, considerando a importância das ferramentas de avaliação da qualidade da assistência para a instituição e a segurança do paciente, este estudo tem como objetivo apresentar evidências científicas sobre as estratégias utilizadas para a avaliação da qualidade da assistência de enfermagem no contexto hospitalar.

\section{Metodologia}

Trata-se de um estudo de revisão integrativa da literatura, desenvolvida com a finalidade de reunir e sintetizar achados de estudos realizados, mediante diferentes metodologias, com o intuito de contribuir para o aprofundamento do conhecimento relativo ao tema investigado (Soares et al., 2014).

Visando manter o rigor científico e metodológico, esta revisão foi alicerçada em protocolo previamente estabelecido e preconizado pelo Joanna Briggs Institute (Peters, Godfrey, McInerney, Soares, Khalil, \& Parker, 2015): 1) Formulação da questão de pesquisa; 2) especificação dos métodos de seleção dos estudos; 3) procedimento de extração dos dados; 4) análise crítica e avaliação dos estudos incluídos 5) extração de dados e 6) apresentação da revisão integrativa do conhecimento 
produzido/publicado. A revisão foi conduzida conforme a metodologia Preferred Reporting Items for Systematic Reviews and Meta-Analyses (PRISMA) (Moher, Liberati, Tetzlaff \& Altman, 2009).

Utilizou-se a estratégia PICo que consiste P (População Alvo) = Avaliação da Qualidade, I (Área de Interesse) $=$ Assistência de Enfermagem, Co (Contexto) = Hospitalar, para a formulação da questão norteadora. Assim a questão norteadora do estudo: "Quais as evidências científicas acerca das estratégias utilizadas para avaliação da qualidade da assistência de enfermagem no ambiente hospitalar?".

A coleta de dados ocorreu nos meses de julho e agosto de 2020 nas bases de dados: Medical Literature Analysis and Retrieval System Online (MEDLINE) via PubMed, Literatura Latino-Americana e do Caribe em Ciências da Saúde (LILACS), Scopus, Cummulative Index to Nursing and Allied Health Literature (CINAHL) e na biblioteca digital Scientific Electronic Library Online (SciELO).

Para garantir o controle de vocabulário e identificação de palavras correspondentes, utilizou-se o Medical Subject Heading Terms (Mesh Terms) e o Descritores em Ciência da Saúde (DeCS). Foram elaborados dois grupos de descritores, estes foram combinados pelo método boleano $O R$ e $A N D$ : (Qualidade Assistencial) AND (Auditoria em Enfermagem) AND (Hospital) AND (Cuidado de enfermagem) AND (Mecanismos de Avaliação da Assistência à Saúde) OR (Software) e (Quality of Care) AND (Nursing audit) AND (Healthcare Evaluation Mechanisms) OR (Software) AND (Hospitals) AND (Nursing Care).

Para a seleção dos dados foram critérios de inclusão: artigos indexados nas bases de dados selecionadas, sem recorte de tempo, nos idiomas português, espanhol e inglês, cujo título e/ou resumo fazem referência à temática pesquisada.

Os critérios de exclusão foram: publicações em forma de teses, dissertações, monografias, livros, carta-resposta, publicados em anais de congressos ou editoriais e artigos que não se relacionassem com o tema.

A avaliação das referências incluídas por meio de três etapas de leitura. Na primeira etapa foram lidos os títulos dos artigos, na segunda foi realizada a leitura dos resumos, e na terceira e última etapa da leitura, foi realizada e avaliação na íntegra dos estudos. Em todas as etapas, a leitura e a análise dos artigos foram realizadas por dois pesquisadores independentes para evitar vieses de seleção, obtendo-se $90 \%$ de concordância entre revisores. Eventuais discordâncias foram discutidas e avaliadas conjuntamente para se estabelecer um consenso.

Na primeira e na segunda leitura foram excluídos os artigos que não atendiam aos critérios de inclusão e exclusão da pesquisa ou que não estavam disponíveis na íntegra. Na terceira leitura, os artigos foram lidos na íntegra e, para se verificar a qualidade e pertinência de sua inclusão. Após a seleção das referências incluídas, para melhor organização e apresentação dos resultados, foram identificadas, traduzidas e transcritas as informações pertinentes e que correspondiam aos elementos de interesse. Com o intuito de sistematizar a informação, os dados extraídos dos estudos foram compilados de forma descritiva em um quadro previamente elaborado, que facilitou a identificação e reformulação das informações.

\section{Resultados}

A amostra da revisão foi composta por 13 estudos. A captação dessas referências está ilustrada na Figura 1. 
Figura 1. Fluxograma de operacionalização para seleção dos estudos incluídos na revisão integrativa.

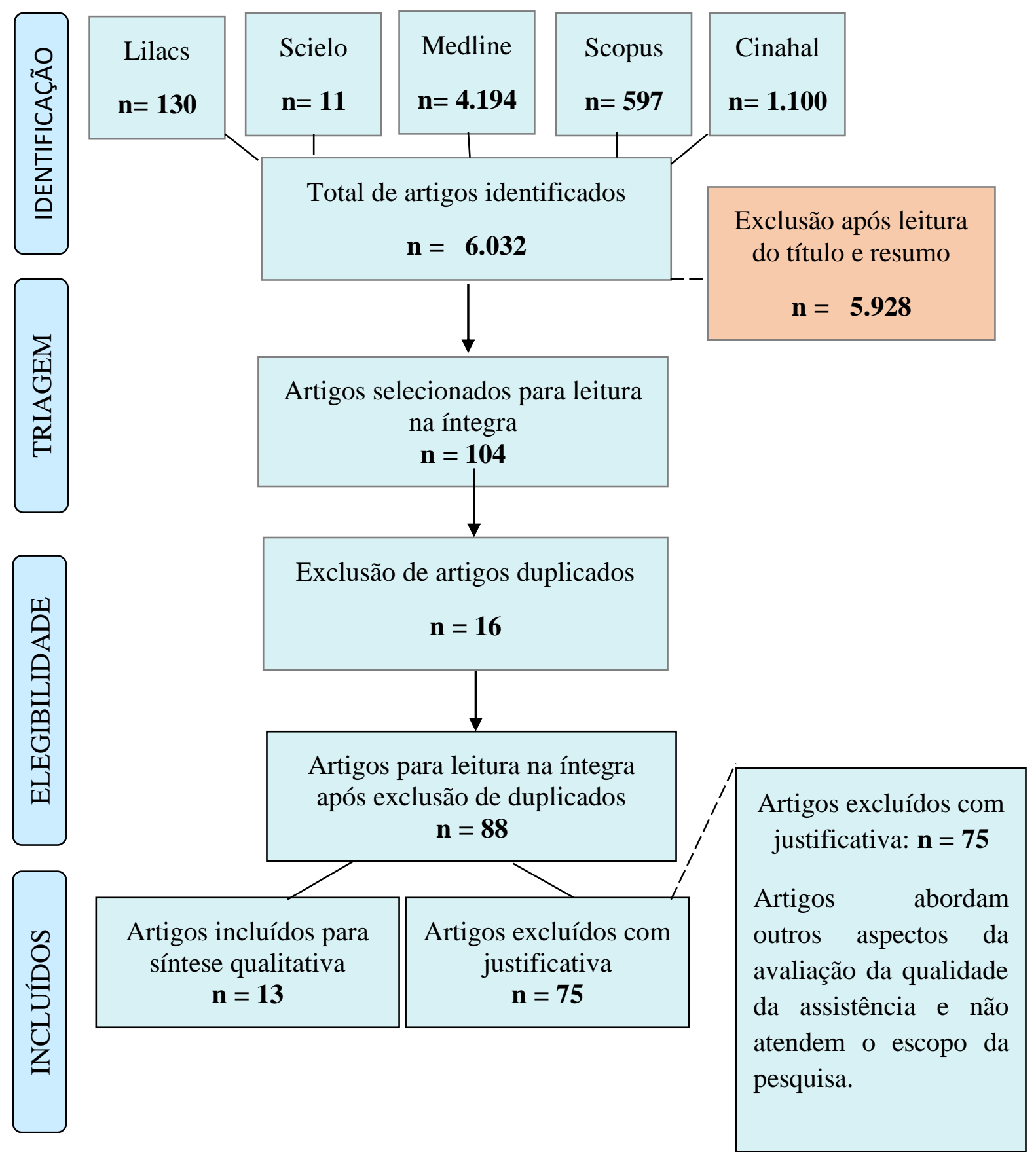

Fonte: Dados obtidos do estudo. Londrina (2020).

Entre os artigos incluídos, onze (84,6\%) estavam redigidos em inglês, um $(7,6 \%)$ em português e um $(7,6 \%)$ em espanhol. Em relação ao país de origem da pesquisa, dois foram desenvolvidos na Suécia, dois da Inglaterra e um artigo em cada país listado a seguir: Brasil, Portugal, Noruega, Sérvia, Japão, Canadá, Colômbia, Turquia e Holanda. Os artigos foram publicados de 1994 a 2018, sendo que os anos de maior número de publicações foram 2005, 2010 e 2012, com dois estudos em cada. Os autores de todos os estudos selecionados são enfermeiros. 
A respeito do método utilizado, nove $(69,2 \%)$ artigos foram realizados com abordagem metodológica e quatro $(30,8 \%)$ com abordagem qualitativa. Quanto ao nível de evidência, devido a temática pesquisada, um (Johanssona, Oleni \& Fridlund, 2005) foi classificado como nível 3 (estudos quase-experimentais); doze (Norman et al, 1994; Laschinger, Hall, Pedersen, \& Almost, 2005; Sjetne \& Stavem, 2006; Vanhaecht, et al, 2007; Haddad \& Évora, 2008; Kobayashi, Takemura \& Kanda, 2011; Ipek Coban \& Kasikci, 2010; Lindgren \& Andersson, 2011; Milutinović, Simin, Brkić \& Brkić, 2012; Amaral, Ferreira \& Lake, 2012; Lenis-Victoria \& Manrique-Abril, 2015; Gillespie \& Reader, 2018) nível 4 (estudos descritivos).

Para favorecer a análise, os resultados estão apresentados em forma de quadro síntese (Quadro 1).

Quadro 1. Caracterização dos estudos selecionados em bases de dados, segundo título, país de origem, ano de publicação, instrumento utilizada e principais resultados.

\begin{tabular}{|c|c|c|c|c|}
\hline Título do Artigo & $\begin{array}{c}\text { Pais/Ano } \\
\text { Publicação }\end{array}$ & Tipo de pesquisa & $\begin{array}{c}\text { Ferramenta } \\
\text { Utilizada }\end{array}$ & Principais Resultados \\
\hline $\begin{array}{c}\text { Evaluation of Kitson's Therapeutic } \\
\text { Nursing Function Matrix in the } \\
\text { assessment of quality of nursing } \\
\text { care in hospital (Norman et al, } \\
\text { 1994) }\end{array}$ & Inglaterra / 1994 & Metodológica & $\begin{array}{c}\text { Therapeutic } \\
\text { Nursing Function } \\
\text { Indicator }(\mathrm{TNFI})\end{array}$ & $\begin{array}{c}\text { TNFI é sensível apenas em } \\
\text { enfermarias médicas e } \\
\text { cirúrgicas. }\end{array}$ \\
\hline $\begin{array}{l}\text { A Psychometric Analysis of the } \\
\text { Patient Satisfaction With Nursing } \\
\text { Care Quality Questionnaire } \\
\text { (Laschinger, Hall, Pedersen, \& } \\
\text { Almost, 2005) }\end{array}$ & Canadá / 2005 & Metodológica & $\begin{array}{l}\text { Patient Satisfaction } \\
\text { with Nursing Care } \\
\text { Quality } \\
\text { Questionnaire } \\
\text { (PSNCQQ) }\end{array}$ & $\begin{array}{l}\text { O instrumento é válido para } \\
\text { avaliar a qualidade da } \\
\text { assistência de enfermagem. }\end{array}$ \\
\hline $\begin{array}{l}\text { Nurses' assessments and patients' } \\
\text { perceptions: development of the } \\
\text { night nursing care instrument } \\
\text { (NNCI), measuring nursing care at } \\
\text { night (Johanssona, Oleni \& } \\
\text { Fridlund, 2005) }\end{array}$ & Suécia / 2005 & Metodológica & $\begin{array}{l}\text { Night Nursing Care } \\
\text { Instrument (NNCI) }\end{array}$ & $\begin{array}{l}\text { O instrumento de cuidado } \\
\text { tem uma confiabilidade } \\
\text { geral aceitável e validade }\end{array}$ \\
\hline $\begin{array}{c}\text { Properties of a Norwegian version } \\
\text { of the Ward Organisational } \\
\text { Features Scales (Sjetne \& Stavem, } \\
\text { 2006) }\end{array}$ & Noruega / 2006 & Metodológica & $\begin{array}{c}\text { Ward } \\
\text { Organisational } \\
\text { Features Scales } \\
\text { (WOFS) }\end{array}$ & $\begin{array}{l}\text { Instrumento confiável e } \\
\text { válido }\end{array}$ \\
\hline $\begin{array}{l}\text { Development and validation of a } \\
\text { care process self-evaluation tool } \\
\quad \text { (Vanhaecht, et al, 2007) }\end{array}$ & $\begin{array}{c}\text { Holanda e } \\
\text { Bélgica / } 2007\end{array}$ & Metodológica & $\begin{array}{l}\text { Care Process Self- } \\
\text { Evaluation Tool } \\
\text { (CPSET) }\end{array}$ & $\begin{array}{l}\text { Instrumento confiável e } \\
\text { válido }\end{array}$ \\
\hline
\end{tabular}




\begin{tabular}{|c|c|c|c|c|}
\hline Título do Artigo & $\begin{array}{l}\text { Pais/Ano } \\
\text { Publicação }\end{array}$ & Tipo de pesquisa & $\begin{array}{c}\text { Ferramenta } \\
\text { Utilizada }\end{array}$ & Principais Resultados \\
\hline $\begin{array}{c}\text { Qualidade da assistência de } \\
\text { enfermagem: a opinião do paciente } \\
\text { internado em hospital universitário } \\
\text { público (Haddad \& Évora, 2008) }\end{array}$ & Brasil / 2008 & Qualitativa & $\begin{array}{l}\text { Roteiro de } \\
\text { Entrevista do } \\
\text { Paciente }\end{array}$ & $\begin{array}{l}\text { Instrumento valioso para } \\
\text { identificar a qualidade da } \\
\text { prática assistencial } \\
\text { executada pela equipe }\end{array}$ \\
\hline $\begin{array}{c}\text { Patient perception of nursing service } \\
\text { quality; an applied model of } \\
\text { Donabedian's structure-process- } \\
\text { outcome approach theory } \\
\text { (Kobayashi, Takemura \& Kanda, } \\
\text { 2011) }\end{array}$ & Japão / 2010 & Metodológica & $\begin{array}{l}\text { Questionário de } \\
\text { Satisfação do } \\
\text { Paciente }\end{array}$ & $\begin{array}{l}\text { Instrumento válido e } \\
\text { confiável }\end{array}$ \\
\hline $\begin{array}{c}\text { Reliability and validity of the scale of } \\
\text { patient perception of hospital } \\
\text { experience with nursing care in a } \\
\text { Turkish population (Ipek Coban \& } \\
\text { Kasikci, 2010) }\end{array}$ & Turquia, 2010 & Metodológica & $\begin{array}{l}\text { Patient Perception } \\
\text { of Hospital } \\
\text { Experience with } \\
\text { Nursing Care } \\
\text { (PPHEN) }\end{array}$ & $\begin{array}{c}\text { Instrumento válido e } \\
\text { Confiável }\end{array}$ \\
\hline $\begin{array}{c}\text { The Karen instruments for } \\
\text { measuring quality of nursing care- } \\
\text { construct validity and internal } \\
\text { consistency (Lindgren \& Andersson, } \\
\text { 2011) }\end{array}$ & Suécia / 2011 & Metodológica & Instrumento Karen & $\begin{array}{l}\text { Os instrumentos Karen- } \\
\text { paciente e Karen-pessoal } \\
\text { alcançou níveis aceitáveis } \\
\text { de validade de construto }\end{array}$ \\
\hline $\begin{array}{c}\text { The patient satisfaction with nursing } \\
\text { care quality: the psychometric study } \\
\text { of the Serbian version of PSNCQ } \\
\text { questionnaire (Milutinović, Simin, } \\
\text { Brkić \& Brkić, 2012) }\end{array}$ & Sérvia / 2012 & Qualitativa & $\begin{array}{l}\text { Patient Satisfaction } \\
\text { Nursing Care } \\
\text { Quality } \\
\text { Questionnaire } \\
\text { (PSNCQQ) }\end{array}$ & $\begin{array}{l}\text { Confiável e valido para } \\
\text { ambientes cirúrgicos }\end{array}$ \\
\hline $\begin{array}{l}\text { Validação da Practice Environment } \\
\text { Scale do Nursing Work Index (PES- } \\
\text { NWI) para a população de } \\
\text { enfermeiros portugueses (Amaral, } \\
\text { Ferreira \& Lake, 2012) }\end{array}$ & Portugal / 2012 & Metodológica & $\begin{array}{c}\text { Practice } \\
\text { Environment Scale } \\
\text { do Nursing Work } \\
\text { Index (PES-NWI) }\end{array}$ & $\begin{array}{l}\text { Confiável e valido para } \\
\text { ambientes hospitalares }\end{array}$ \\
\hline $\begin{array}{l}\text { Calidad del cuidado de enfermería } \\
\text { percibida por pacientes } \\
\text { hospitalizados (Lenis-Victoria \& } \\
\text { Manrique-Abril, 2015) }\end{array}$ & $\begin{array}{l}\text { Colômbia / } \\
2015\end{array}$ & Qualitativa & $\begin{array}{l}\text { Cuestionario de } \\
\text { Calidad del } \\
\text { Cuidado de } \\
\text { Enfermería } \\
\text { (CUCACE) }\end{array}$ & $\begin{array}{l}\text { Instrumento confiável e } \\
\text { válido }\end{array}$ \\
\hline $\begin{array}{c}\text { Patient-Centered Insights_Using } \\
\text { Health Care Complaints to Reveal } \\
\text { Hot Spots and Blind Spots in Quality } \\
\text { and Safety (Gillespie \& Reader, } \\
\text { 2018) }\end{array}$ & Inglaterra / 2018 & Qualitativa & $\begin{array}{l}\text { Ferramenta de } \\
\text { Análise de } \\
\text { Reclamações de } \\
\text { Saúde (HCAT) }\end{array}$ & $\begin{array}{l}\text { Reclamações de cuidados } \\
\text { de saúde podem ser usadas } \\
\text { para identificar pontos } \\
\text { quentes e cegos de } \\
\text { atendimento inseguro e de } \\
\text { baixa qualidade }\end{array}$ \\
\hline
\end{tabular}

Fonte: Autores.

Com vistas a otimizar a avaliação, as ferramentas encontradas nos estudos estão apresentadas em forma de quadro síntese (Quadro2). 
Quadro 2. Caracterização das ferramentas encontradas segundo nome, estrutura e finalidade da ferramenta. * Ferramenta descrita em mais de um artigo.

\begin{tabular}{|c|c|c|}
\hline $\begin{array}{c}\text { Ferramenta de avaliação da } \\
\text { qualidade }\end{array}$ & $\begin{array}{l}\text { Estrutura da } \\
\text { Ferramenta }\end{array}$ & dade \\
\hline $\begin{array}{c}\text { Therapeutic Nursing Function } \\
\text { Indicator (TNFI) (Norman et al, } \\
\text { 1994) }\end{array}$ & $\begin{array}{l}\text { Questionário / } \\
26 \text { itens }\end{array}$ & $\begin{array}{l}\text { Avalia a forma como o enfermeiro define o cuidado, a } \\
\text { proporção de tempo despendido em atividades de cuidados, } \\
\text { gestão ativa na enfermaria e outros fatores adicionais } \\
\text { considerados importantes. }\end{array}$ \\
\hline $\begin{array}{c}\text { Patient Satisfaction with } \\
\text { Nursing Care Quality } \\
\text { Questionnaire (PSNCQQ)* } \\
\text { (Milutinović, Simin, Brkić \& } \\
\text { Brkić, 2012) }\end{array}$ & $\begin{array}{l}\text { Questionário / } \\
22 \text { itens }\end{array}$ & $\begin{array}{l}\text { Avalia os cuidados diários, equipe e ambiente hospital, } \\
\text { informações, admissões, qualidade geral de cuidados. }\end{array}$ \\
\hline $\begin{array}{l}\text { Night Nursing Care Instrument } \\
\text { (NNCI) (Johanssona, Oleni \& } \\
\text { Fridlund, 2005) }\end{array}$ & $\begin{array}{l}\text { Questionário / } \\
11 \text { itens }\end{array}$ & $\begin{array}{l}\text { Avalia as intervenções de enfermagem com os pacientes } \\
\text { durante o período noturno. }\end{array}$ \\
\hline $\begin{array}{l}\text { Ward Organisational Features } \\
\text { Scales (WOFS) (Sjetne \& } \\
\text { Stavem, 2006) }\end{array}$ & $\begin{array}{l}12 \text { escalas } \\
\text { agrupadas }\end{array}$ & $\begin{array}{l}\text { Avalia } 6 \text { domínios: ambiente físico da enfermaria, prática } \\
\text { profissional de enfermagem, prática de liderança da } \\
\text { enfermeira, relações entre a equipe de enfermagem, inter- } \\
\text { relações profissionais e controle e resolução de problemas }\end{array}$ \\
\hline $\begin{array}{l}\text { Care Process Self-Evaluation } \\
\text { Tool (CPSET) (Vanhaecht, et } \\
\text { al, 2007) }\end{array}$ & $\begin{array}{l}\text { Questionário / } \\
29 \text { itens }\end{array}$ & $\begin{array}{l}\text { Tem o objetivo de definir as características chave do processo } \\
\text { de atendimento que estão tendo um impacto na organização. }\end{array}$ \\
\hline $\begin{array}{l}\text { Roteiro de Entrevista do } \\
\qquad \text { Paciente } \\
\text { (Haddad \& Évora, 2008) }\end{array}$ & & $\begin{array}{l}\text { Avaliar a percepção do paciente acerca da qualidade da } \\
\text { assistência de enfermagem de acordo com as necessidades } \\
\text { humanas básicas }\end{array}$ \\
\hline $\begin{array}{l}\text { Questionário de Satisfação do } \\
\text { Paciente (Kobayashi, Takemura } \\
\text { \& Kanda, 2011) }\end{array}$ & $\begin{array}{l}37 \text { itens. } 14 \\
\text { domínios }\end{array}$ & $\begin{array}{l}\text { Representar as percepções de pacientes internados } \\
\text { relacionadas à estrutura, processo e resultados }\end{array}$ \\
\hline $\begin{array}{l}\text { Patient Perception of Hospital } \\
\text { Experience with Nursing Care } \\
\text { (PPHEN) (Ipek Coban \& } \\
\text { Kasikci, 2010) }\end{array}$ & 15 itens & $\begin{array}{l}\text { Avaliar a qualidade do cuidado a partir da satisfação do } \\
\text { paciente }\end{array}$ \\
\hline $\begin{array}{l}\text { Karen-patient e Karen- } \\
\text { personnel (Lindgren \& } \\
\quad \text { Andersson, 2011) }\end{array}$ & 35 itens & $\begin{array}{l}\text { Avaliação da percepção do paciente quanto à qualidade do } \\
\text { atendimento, fatores que influenciam a qualidade, aspectos } \\
\text { importantes do cuidado, os objetivos da assistência de } \\
\text { enfermagem, a qualidade da assistência relacionada ao } \\
\text { autocuidado e fatores que aumentam a qualidade do } \\
\text { atendimento }\end{array}$ \\
\hline Cuestionario de Calidad del & Questionário / & Avalia a experiência e satisfação do paciente com os cuidados \\
\hline
\end{tabular}


Research, Society and Development, v. 10, n. 2, e45310212679, 2021

(CC BY 4.0) | ISSN 2525-3409 | DOI: http://dx.doi.org/10.33448/rsd-v10i2.12679

\begin{tabular}{|c|c|l|}
\hline $\begin{array}{c}\text { Cuidado de Enfermería } \\
\text { (CUCACE) (Lenis-Victoria \& } \\
\text { Manrique-Abril, 2015) }\end{array}$ & 45 itens & de enfermagem \\
\hline $\begin{array}{c}\text { Ferramenta de Análise de } \\
\text { Reclamações de Saúde (HCAT) } \\
\text { (Gillespie \& Reader, 2018) }\end{array}$ & 3 domínios & Classificar e analisar as reclamações realizadas \\
\hline $\begin{array}{c}\text { Practice Environment Scale do } \\
\text { Nursing Work Index (PES- } \\
\text { NWI) (Amaral, Ferreira \& }\end{array}$ & Questionário / & \\
Lake, 2012) & 31 itens & Descrever e avaliar características da pratica assistencial de \\
enfermagem em ambientes hospitalares
\end{tabular}

Fonte: Autores.

No que se refere as ferramentas utilizadas para avaliar a qualidade da assistência de enfermagem, houve predominância de questionários construídos e validados para analisar diversas variáveis do cuidado. Entre eles, seis (50\%) avaliam aspectos relacionados a satisfação dos pacientes com a assistência de enfermagem, quatro (33,4\%) avaliam características do processo de cuidado, um $(8,3 \%)$ avalia aspectos de intervenções de enfermagem e um $(8,3 \%)$ avalia aspectos relacionados a reclamações dos pacientes. A ferramenta Patient Satisfaction with Nursing Care Quality Questionnaire $(P S N C Q Q)$ foi a única descrita em mais de um artigo.

\section{Discussão}

Os artigos desta revisão apresentam contribuições de diferentes ferramentas para a avaliação da qualidade da assistência de enfermagem. A avaliação é um dos principais mecanismos do controle da qualidade e é debatida nos cenários da saúde há anos (Miguel \& Salomi, 2004; Hovenga \& Lowe, 2020). Mesmo com o conhecimento de sua importância e da busca frequente dos serviços pela melhoria da qualidade, observa-se, a partir dos resultados encontrados, que ao longo dos anos não houve aumento no número de publicações sobre o desenvolvimento e atualização de ferramentas utilizadas na avaliação da qualidade da assistência. A baixa produção cientifica sobre o tema vai ao encontro do descrito em outros estudos, onde relatam que as principais medidas de desempenho do sistema de saúde não mudam por décadas (Braithwaite, 2018).

Pouco abordada mundialmente, observou-se que a maior parte dos estudos encontrados foram realizados em continente europeu e isso pode estar diretamente relacionado a qualidade dos sistemas de saúde e da cultura de segurança do paciente e de qualidade da assistência nos países. Na Suécia, por exemplo, a legislação obriga os profissionais de saúde a acompanhar e documentar continuamente a qualidade do atendimento (Lindgren \& Andersson, 2011).

Outro importante aspecto observado é o fato de que entre as ferramentas utilizadas nos estudos, houve a predominância de questionários. Isso revela que apesar da crescente tendência de inovações tecnológicas na saúde e enfermagem, pouco se evoluiu no que se refere ao tema "avaliação de qualidade". Durante seu processo de trabalho, o enfermeiro tem tido diversas aproximações com a tecnologia (Grossi, Pisa \& Marin, 2015) porém, por falta de divulgação, a área ainda é pouco explorada e raros estudos são conduzidos para verificar potenciais usos, avaliações do que tem sido aplicado e perspectivas de novos desenvolvimentos de sistemas que possam apoiar a prática (Grossi, Pisa \& Marin, 2015).

A segurança, eficácia, o cuidado centrado na pessoa, a acessibilidade, a pontualidade, a eficiência e a equidade são as dimensões mais conhecidas para indicar a qualidade dos serviços de enfermagem (National Academies of Sciences, Engineering, and Medicine et al., 2018). Visto isso, a qualidade pode ser aferida a partir de diferentes perspectivas. 
Os artigos selecionados para a revisão utilizaram os aspectos relacionados a satisfação dos pacientes com a assistência de enfermagem, as características do processo de cuidado, os aspectos de intervenções de enfermagem e os aspectos relacionados às reclamações dos pacientes.

Quanto aos instrumentos que analisam os aspectos relacionados à satisfação do paciente, os estudos explicitaram que a satisfação do paciente com o cuidado de enfermagem está diretamente relacionada à percepção subjetiva que o indivíduo tem sobre o cuidado que recebe (Haddad \& Evora, 2008) e sua mensuração é importante, pois são esses cuidados que geralmente determinam a experiências do paciente com o hospital (Ipek Coban \& Kasikci, 2010). Os pacientes estão satisfeitos apenas quando suas necessidades básicas são atendidas (Kobayashi, Takemura \& Kanda, 2011; Lindgren \& Andersson, 2011). Além do cuidado em si, o grau de satisfação ou de insatisfação pode decorrer das relações interpessoais entre o profissional e o paciente (Haddad \& Evora, 2008).

Foi demonstrado que relacionar a satisfação do paciente com a avaliação de outros aspectos do cuidado, da equipe e das e intervenções de enfermagem pode trazer ainda mais resultados na mensuração da qualidade da assistência. (Lindgren \& Andersson, 2011; Lenis-Victoria \& Manrique-Abril, 2015).

No geral, todos os artigos avaliaram que os instrumentos que utilizam a satisfação do paciente como medida são valiosos para identificar a qualidade da prática assistencial executada pela equipe (Haddad \& Evora, 2008; Ipek Coban \& Kasikci, 2010; Kobayashi, Takemura \& Kanda, 2011; Lindgren \& Andersson, 2011; Lenis-Victoria \& Manrique-Abril, 2015).

Os estudos que discutem os instrumentos que analisam os aspectos relacionados as características do processo de cuidado, sugeriram que avaliar presença ou não do conjunto de características organizacionais no ambiente hospitalar que sustentam a prática profissional de enfermagem é valido para uma avaliação da qualidade adequada. Tais ferramentas permitem que o avaliador identifique as características principais de uma instituição bem organizada e por isso também são ótimas opções para serem utilizadas na avaliação da qualidade da assistência (Norman et al, 1994; Sjetne \& Stavem, 2006; Vanhaecht et al, 2007; Amaral, Ferreira \& Lake, 2012).

No instrumento que analisa os aspectos relacionados às intervenções de enfermagem foram utilizadas escalas de avaliação da percepção dos pacientes e equipe de enfermagem sobre as intervenções que são realizadas no período noturno. Após a coleta, os dados foram cruzados e indicaram qualidade. As divergências encontradas por meio desta avaliação demonstram aos avaliadores os pontos assistenciais que precisam ser reforçados (Johansson, Oléni \& Fridlund, 2005).

Quanto ao instrumento que analisa os aspectos relacionados às reclamações dos pacientes, trata-se de uma ferramenta que conceitua hierarquicamente os problemas relatados e os classifica usando sete tipos de problemas dividido em três domínios. Após os testes realizados, concluiu-se que as reclamações de cuidados de saúde podem sim, ser usadas para identificar pontos quentes e pontos cegos de atendimento inseguro e de baixa qualidade, proporcionando as instituições novas oportunidades para monitoramento centrado no paciente e aprendizagem organizacional (Gillespie \& Reader, 2018).

Após a avaliação das ferramentas, observou-se que poucas limitações foram pontuadas, nenhuma delas relacionada a aplicação das ferramentas. Dos artigos inclusos, alguns possuíam uma população restrita ao estudo, como quantidade reduzida de participantes, sendo necessários estudos aplicados em populações mais abrangentes para comprovar se as ferramentas realmente são eficazes.

\section{Considerações Finais}

Com base no estudo realizado é possível concluir que as ferramentas de avaliação da qualidade da assistência são tão importantes para as instituições quanto para os pacientes. E apesar da sua relevância elas são pouco estudadas e do ponto de vista tecnológico, pouco tem evoluído. Os questionários aqui pontuados avaliam vários aspectos do cuidado e ainda são as ferramentas mais utilizadas para tal finalidade. 
Os resultados desta pesquisa buscam apresentar uma atualização sobre a temática abordada e recomendar mais estudos na área de Enfermagem voltados para as ferramentas de avaliação da qualidade da assistência.

\section{Referências}

Amaral, A. F. S., Ferreira, P. L., \& Lake, E. (2012). Validation of the Practice Environment Scale of the Nursing Work Index (PES-NWI) for the Portuguese nurse population. International Journal of Caring Sciences, 5(3), 280-288.

Braithwaite, J. (2018). Changing how we think about healthcare improvement. bmj, 361.

Gillespie, A., \& Reader, T. W. (2018). Patient-centered insights: using health care complaints to reveal hot spots and blind spots in quality and safety. The Milbank Quarterly, 96(3), 530-567.

Haddad, M. D. C. L., \& Évora, Y. D. M. (2008). Qualidade da assistência de enfermagem: a opinião do paciente internado em hospital universitário público. Ciência, Cuidado e Saúde, 7, 45-52.

Hovenga, E. J. S., \& Lowe, C. (2020). Measuring health service quality. Measuring Capacity to Care Using Nursing Data, 355-388.

Ipek Coban, G., \& Kasikci, M. (2010). Confiabilidade e validade da escala de percepção do paciente sobre a experiência hospitalar com cuidados de enfermagem em uma população turca. Journal of Clinical Nursing, 19 (13-14), 1929-1934.

Johansson, P., Oléni, M., \& Fridlund, B. (2005). Nurses' assessments and patients' perceptions: development of the night nursing care instrument (NNCI), measuring nursing care at night. International Journal of Nursing Studies, 42(5), 569-578.

Karaca, A., \& Durna, Z. (2019). Patient satisfaction with the quality of nursing care. Nursing open, 6(2), 535-545.

Kobayashi, H., Takemura, Y., \& Kanda, K. (2011). Patient perception of nursing service quality, an applied model of Donabedian's structure-process-outcome approach theory. Scandinavian journal of caring sciences, 25(3), 419-425.

Laschinger, H. S., Hall, L. M., Pedersen, C., \& Almost, J. (2005). A psychometric analysis of the patient satisfaction with nursing care quality questionnaire: an actionable approach to measuring patient satisfaction. Journal of nursing care quality, 20(3), 220-230.

Lenis-Victoria, C. A., \& Manrique-Abril, F. G. (2015). Calidad del cuidado de enfermería percibida por pacientes hospitalizados. Aquichan, 15(3), 413-425.

Lindgren, M., \& Andersson, I. S. (2011). The Karen instruments for measuring quality of nursing care: construct validity and internal consistency. International journal for quality in health care, 23(3), 292-301.

Lindgren, M., \& Andersson, I. S. (2011). The Karen instruments for measuring quality of nursing care: construct validity and internal consistency. International journal for quality in health care, 23(3), 292-301.

Marin, H. F., Grossi, L. M., \& Pisa, I. T. (2015). Tecnologia da Informação e comunicação na auditoria em Enfermagem. Journal of Health Informatics, 7(1).

Miguel, P. A. C., \& Salomi, G. E. (2004). Uma revisão dos modelos para medição da qualidade em serviços. Production, 14(1), 12-30.

Milutinović, D., Simin, D., Brkić, N., \& Brkić, S. (2012). The patient satisfaction with nursing care quality: the psychometric study of the Serbian version of PSNCQ questionnaire. Scandinavian journal of caring sciences, 26(3), 598-606.

Moher, D., Liberati, A., Tetzlaff, J., Altman, DG, \& Prisma Group. (2009). Itens de relatório preferidos para revisões sistemáticas e meta-análises: a declaração PRISMA. Medicamento PLoS, 6 (7), e1000097.

National Academies of Sciences, Engineering, and Medicine. (2018). Crossing the global quality chasm: Improving health care worldwide.

Norman, I. J. et al., Evaluation of Kitson's therapeutic nursing function matrix in the assessment of quality of nursing care in hospital. International journal of nursing studies, 31(4), 337-348.

Peters, M., Godfrey, C., McInerney, P., Soares, C., Khalil, H., \& Parker, D. (2015). The Joanna Briggs Institute reviewers' manual 2015: methodology for JBI scoping reviews.

Recio-Saucedo, A., Dall'Ora, C., Maruotti, A., Ball, J., Briggs, J., Meredith, P., \& Griffiths, P. (2018). What impact does nursing care left undone have on patient outcomes? Review of the literature. Journal of Clinical Nursing, 27(11-12), 2248-2259.

Richards, D. A., Hilli, A., Pentecost, C., Goodwin, V. A., \& Frost, J. (2018). Fundamental nursing care: A systematic review of the evidence on the effect of nursing care interventions for nutrition, elimination, mobility and hygiene. Journal of clinical nursing, 27(11-12), 2179-2188.

Rocha, E. S. B., Trevizan, M. A., Júnior, K. R., \& Castelhano-Souza, M. (2013). Gestão da qualidade na enfermagem brasileira: revisão de literatura [Quality management in brazilian nursing: a literature review]. Revista Enfermagem UERJ, 21(6), 812-817.

Santos, C. M. D. C., Pimenta, C. A. D. M., \& Nobre, M. R. C. (2007). A estratégia PICO para a construção da pergunta de pesquisa e busca de evidências. Revista Latino-Americana de Enfermagem, 15(3), 508-511.

Sjetne, I.S., Stavem, K., Properties of a Norwegian version of the Ward Organisational features scales. Scandinavian journal of caring sciences, 20(4), 455$461,2006$. 
Research, Society and Development, v. 10, n. 2, e45310212679, 2021

(CC BY 4.0) | ISSN 2525-3409 | DOI: http://dx.doi.org/10.33448/rsd-v10i2.12679

Soares, C. B., Hoga, L. A. K., Peduzzi, M., Sangaleti, C., Yonekura, T., \& Silva, D. R. A. D. (2014). Integrative review: concepts and methods used in nursing. Revista da Escola de Enfermagem da USP, 48(2), 335-345.

Vanhaecht, K. et al. Development and validation of a care process self-evaluation tool. Health Services Management Research, 20(3), 189-202. 